\title{
Original
}

\section{Percutaneous Needle Biopsy of the Thorax Using Cone-beam CT Equipped with Flat Panel Detector DSA}

\author{
Shu Takaya ${ }^{1)}$, Toshi Hashimoto ${ }^{2)}$, Minoru Honda ${ }^{1)}$, \\ Noritaka SEINO $^{1)}$, Takashi HashizUme ${ }^{1)}$ and Takehiko GoKAN ${ }^{1)}$
}

\begin{abstract}
This study investigated the method and clinical value of percutaneous needle biopsy of the thoracic region using a combination of cone-beam computed tomography (CBCT) equipped with a flat panel detector for digital subtraction angiography (DSA) and fluoroscopy. In 77 patients with a total of 79 lesions in the thoracic region (67 in the lung field, 6 in the mediastinum, and 6 in the chest wall), CT-like images of the lesions were acquired by $\mathrm{CBCT}$, and needle biopsy was performed with fluoroscopic guidance. Puncture of the mass via the planned route was possible in all cases, and specimens of a sufficient size for pathological diagnosis could be biopsied. Only slight adjustments of the puncture site and angle were made under fluoroscopy. The final diagnosis of the biopsied specimen was malignant disease in 70 of the 77 patients $(90.9 \%)$; 54 of the 77 patients $(70.1 \%)$ had primary lung cancer and in 4 of the 77 patients $(5.2 \%)$ had metastatic tumor. The histological type of the primary lung cancer was adenocarcinoma in 26 of the 77 patients $(33.8 \%)$, squamous cell carcinoma in 22 of the 77 patients $(28.6 \%)$, and small cell carcinoma in 6 of the 77 patients $(7.8 \%)$. Complications occurred in 31 of the 77 patients $(40.3 \%)$, comprising pneumothorax and pulmonary hemorrhage, but no fatal complications such as air embolism or the dissemination of malignant cells eventuated. In addition, unlike CT-guided biopsy, the described procedure requires only an angiography room. This method enables reliable biopsy using 3-dimensional data, and is a promising percutaneous biopsy method.
\end{abstract}

Key words : needle biopsy, thoracic region, CBCT, CT-like images

\section{Introduction}

Percutaneous lung biopsy is generally performed with guidance by fluoroscopy, ultrasonography, and computed tomography (CT). However, cross-sectional views cannot be imaged by fluoroscopy, while ultrasonography is not applicable for lung lesions which are not in contact with the chest wall. CT-guided biopsy is useful for the objective and accurate imaging of the relationship with the surrounding organs, as initially reported by Hagga and

\footnotetext{
1) Department of Radiology, Showa University School of Medicine,, 1-5-8 Hatanodai, Shinagawa-ku, Tokyo 142-8666, Japan.

2) Department of Radiology, Showa University Fujigaoka Hospital.
} 
Alfidi. in $1976^{1)}$. However, unlike ultrasonographic guidance, real-time images cannot be acquired by $\mathrm{CT}$, preventing real-time observation of the needle tip and target region during needle advancing. Thus, biopsy of the thoracic and upper abdominal regions, which move with respiration, was difficult. In Japan, Katada et $a l^{2)}$ and Kato et $a l^{3)}$ first applied CT fluoroscopy-guided biopsy clinically in 1996, although the procedure level still has several disadvantages. Compared to CT-guided biopsy, the exposure level is higher, the instrument is expensive, and a CT room is necessary.

Recent advances in technology now enable the preparation of CT-like images from conebeam data acquired using cone-beam CT (CBCT) equipped with a flat panel detector for digital subtraction angiography (DSA). Planning a 3-dimensional puncture line by CT has been difficult because CT generates data on stacked images. In contrast, CBCT generates volumetric data, thus a 3-dimensional puncture line can be planned using a work station. In addition, the puncture direction can be observed from any angle, and the puncture can be performed following that angle.

Our facility has been performing percutaneous biopsy using CBCT equipped with flat panel detector DSA since 2005. We have found that biopsy can be performed more safely and accurately by acquiring CT-like images of lesions by CBCT and combining it with fluoroscopy, compared to CT-guided biopsy. This study investigated this method and its clinical usefulness for biopsies of the thoracic region.

\section{Subjects}

The study analyzed 79 lesions (67 in the lung field, 6 in the mediastinum, and 6 in the chest wall) in 77 consecutive patients who underwent biopsy using CBCT equipped with a flat panel detector DSA from May 7, 2007 to August 25, 2008. There were 55 male and 22 female patients aged 27-89 years (mean; 68.1 years). The objective of biopsy was to pathologically evaluate abnormal findings on previous imaging diagnoses.

Chest radiographs and CT images were obtained prior to this biopsy in all 77 patients. Bronchoscopy was performed prior to the biopsy in the 67 lung-field lesions. Previously acquired CT images were reviewed with attendant physicians before biopsy in all patients, to confirm that the puncture site and posture could be maintained during biopsy and that there was no hemorrhagic tendency.

\section{Methods}

The patient was placed in the supine or prone position, and a non-X-ray-penetrable marker was fixed to the planned skin puncture site. Volumetric data were collected with 5- or 10-second acquisition periods, 200-degree rotation, 70-KV voltage, and 200-300-mA current, using CBCT equipped with flat panel detector DSA, AXIOM Artis dTA DynaCT (Siemens-Asahi Medical Technologies, Ltd, Tokyo,). A 3-dimensional plan of the skin surface was designed to determine the puncture route, encompassing distance between the 
puncture site and lesion and the puncture angle (Fig. 1-1). Previous CT images were also viewed to plan the puncture route. After the puncture site was marked with a temporary magic ink pen, the skin surface was disinfected and a local anesthetic agent administered.

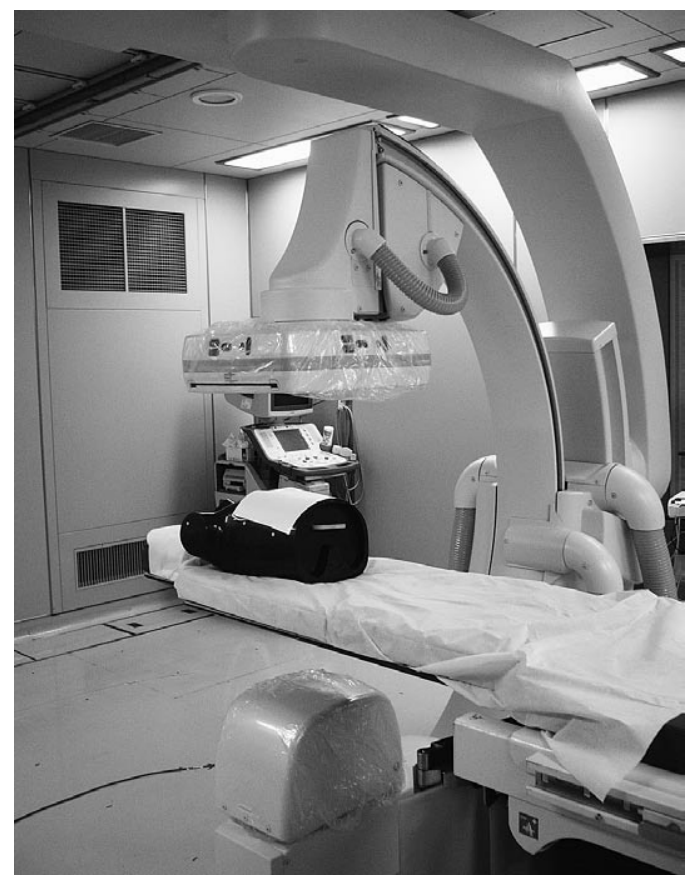

1: AXIOM Artis dTA DynaCT

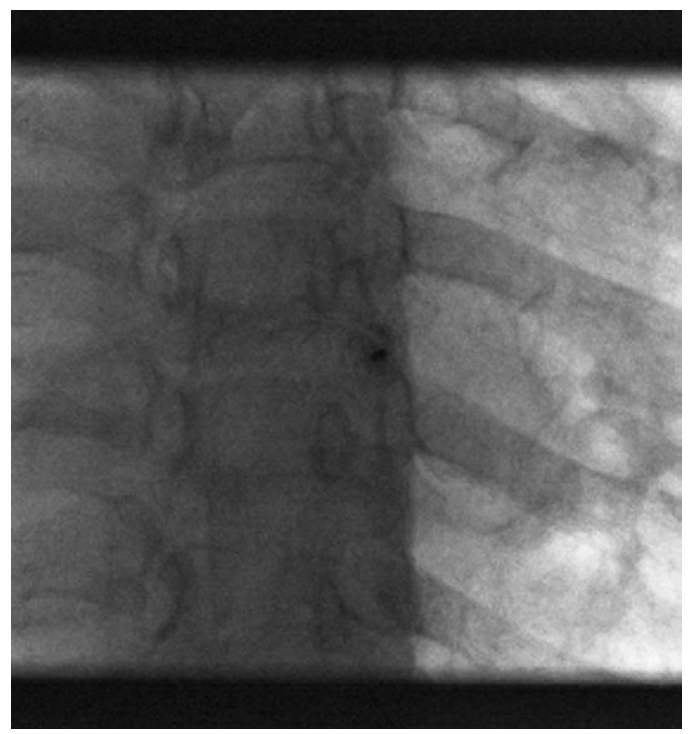

3 : Fluoroscopy image for planning biopsy

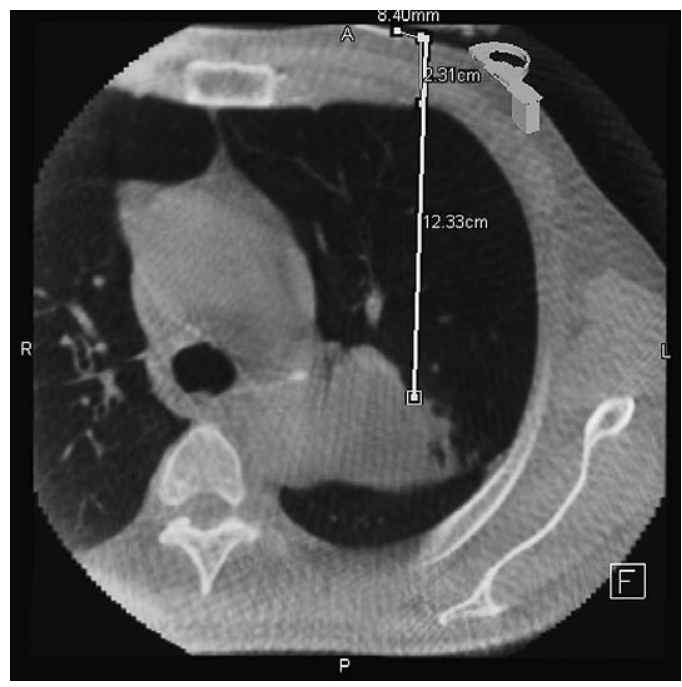

2: Axial CBCT image for planning biopsy

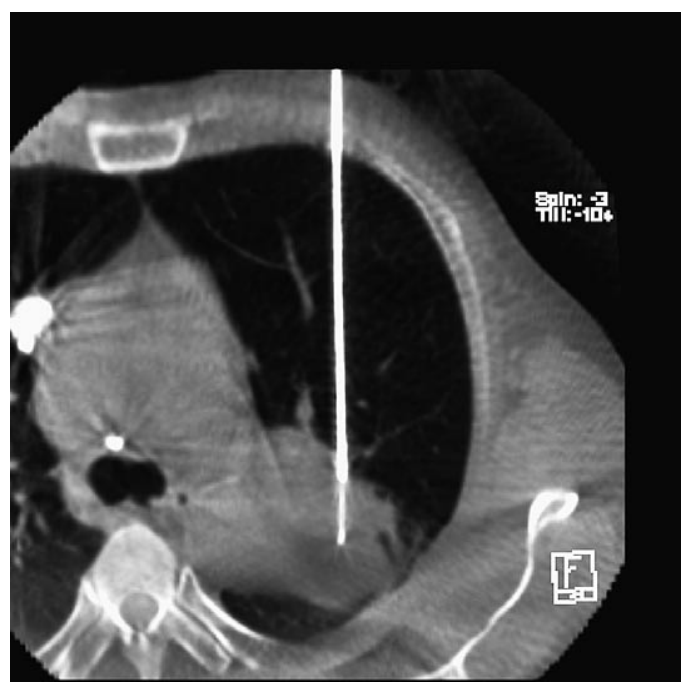

4: Axial CBCT image with needle placement

Fig. 1. 
The biopsy was performed using a Coaxial Quick Needle Biopsy set (Cook Inc., Bloomington, IW USA). A 19-G sheath for the coaxial system was inserted during exhalation, and the skin puncture site was positioned in the center of the fluoroscopic image to reflect the planned puncture angle on the $\mathrm{C}$-arm, so as to arrange the puncture needle in a straight line toward the lesion on fluoroscopy (Fig. 1-2). The needle was advanced toward the measured site with fluoroscopic guidance, until the planned depth was reached. Confirmation and correction of the needle tip position and puncture were then possible by rotating the $\mathrm{C}$-arm. The positions of the sheath and lesion were reconfirmed by CBCT to ensure the absence of problems, and a trocar needle $(20 \mathrm{G})$ was advanced to biopsy the target lesion (Fig. 1-3). CBCT was acquired again to check for potential complications, such as pneumothorax and pulmonary hemorrhage, and then the procedure was completed. The success rate, biopsied specimen, operation time, lesion size, complication rate, and type / level of complications were recorded for analysis.

The following account exemplifies the biopsy method used in this study. A 60-yearold male underwent lung biopsy for pathological examination of a mass in contact with the major fissure of the left upper lobe of the lung. Since puncture from the dorsal side would twice penetrate the pleura, once at the puncture site and again at the pleura between the upper and lower lobes, increasing the frequency of pneumothorax, we selected the anterior side. An oblique upward puncture was planned to ensure sufficient specimen for pathological examination with a puncture that was parallel to the cross-section, based on the relationship between the longitudinal diameter of the tumor and puncture needle on CBCT (Fig. 2-1).

A coaxial needle sheath (19 G) was inserted approximately $2 \mathrm{~cm}$ from the superior bor-

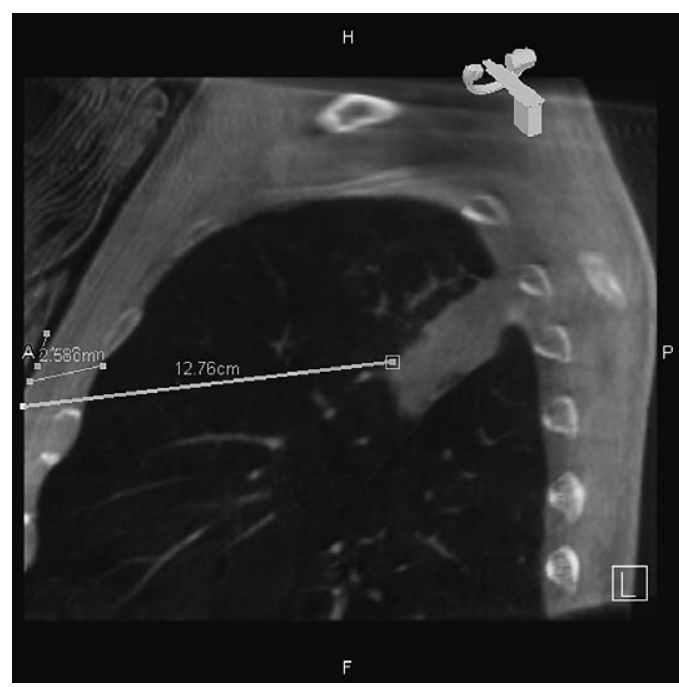

1: Sagittal CBCT image for planning of needle biopsy

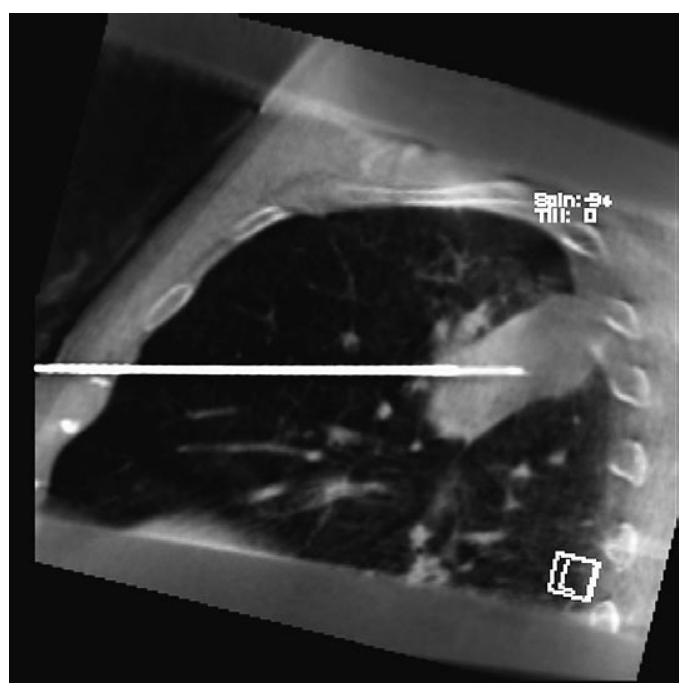

2: Sagittal CBCT image with biopsy needle placement

Fig. 2. 
der of the third rib in the left precordial region, and the needle was placed in the center of the fluoroscopic image directed on a straight line toward the lesion. With fluoroscopic guidance, the sheath was advanced to the planned depth. After confirming that the sheath was present immediately before the lesion by CBCT, the needle $(20 \mathrm{G})$ was advanced, and penetration of the center of the mass by the biopsy needle was confirmed by CBCT (Fig. $2-2$ ), followed by multiple biopsies. Sufficient tissue was obtained from this patient for diagnosis using the regime described, indicating the usefulness of 3-dimensional-guided puncture.

\section{Results}

In all consecutive cases of CT-guided biopsy using CBCT equipped with a flat panel detector DSA, puncture of the mass via the target route was possible, and a specimen of sufficient size for pathological diagnosis could be biopsied. Only slight adjustments of the puncture site and angle were made under fluoroscopy.

The biopsied specimens showed malignant disease by this method in 70 of the 77 patients (90.9\%) ; primary lung cancer in 54 of the 77 patients $(70.1 \%)$ and metastatic tumor in 4 of the 77 patients $(5.2 \%)$. The histological type of the primary lung cancer was adenocarcinoma in 26 of the 77 patients $(33.8 \%)$, squamous cell carcinoma in 22 of the 77 patients $(28.6 \%)$, and small cell carcinoma in 6 of the 77 patients $(7.8 \%)$. The operation time (from the initiation of imaging to confirmation of complications by imaging) was $56.6 \pm 23.6$ minutes (25-139 minutes).

The mean major and minor tumor axes were $43.1 \pm 30.6(12-170)$ and $30.4 \pm 22.7 \mathrm{~mm}$ (10-160 mm), respectively. Taking these values as the 'main diameter', the minimum and maximum main diameters were 11 and $165 \mathrm{~mm}$, respectively, and a main diameter of 15$20 \mathrm{~mm}$ was the most frequent lesion size. Those lesions with a main diameter of $30-\mathrm{mm}$ or less accounted for approximately half $(46.2 \%)$ of the lesions. The mean distance of the mass from the body surface was $46.5 \pm 20.9 \mathrm{~mm}(17-112 \mathrm{~mm})$.

Complications occurred in 31 of the 77 patients $(40.3 \%)$, comprising pneumothorax and pulmonary hemorrhage. No potentially fatal complications were encountered, such as air embolism and the dissemination of malignant cells. Pneumothorax developed in 28 cases of the 77 patients $(36.4 \%)$, but $3 / 4$ of these resolved under course observation alone, and a chest drainage tube was only needed for 7 lesions of the 77 patients (9.1\%). Pulmonary emphysema was noted in $61 \%$ of cases complicated by pneumothorax, but only in $39 \%$ of cases without pneumothorax. Lesion size, distance from the puncture site, and incidence of pneumothorax are shown in Fig. 3. Lesions with a main diameter of $20 \mathrm{~mm}$ or less were often complicated by pneumothorax (about 70\%), and this incidence increased with distance from the puncture site to a relatively high incidence when the distance exceeded $40 \mathrm{~mm}$. Pulmonary hemorrhage occurred in 5 of the 77 patients $(6.5 \%)$, but all were mild and required no specific treatment. 


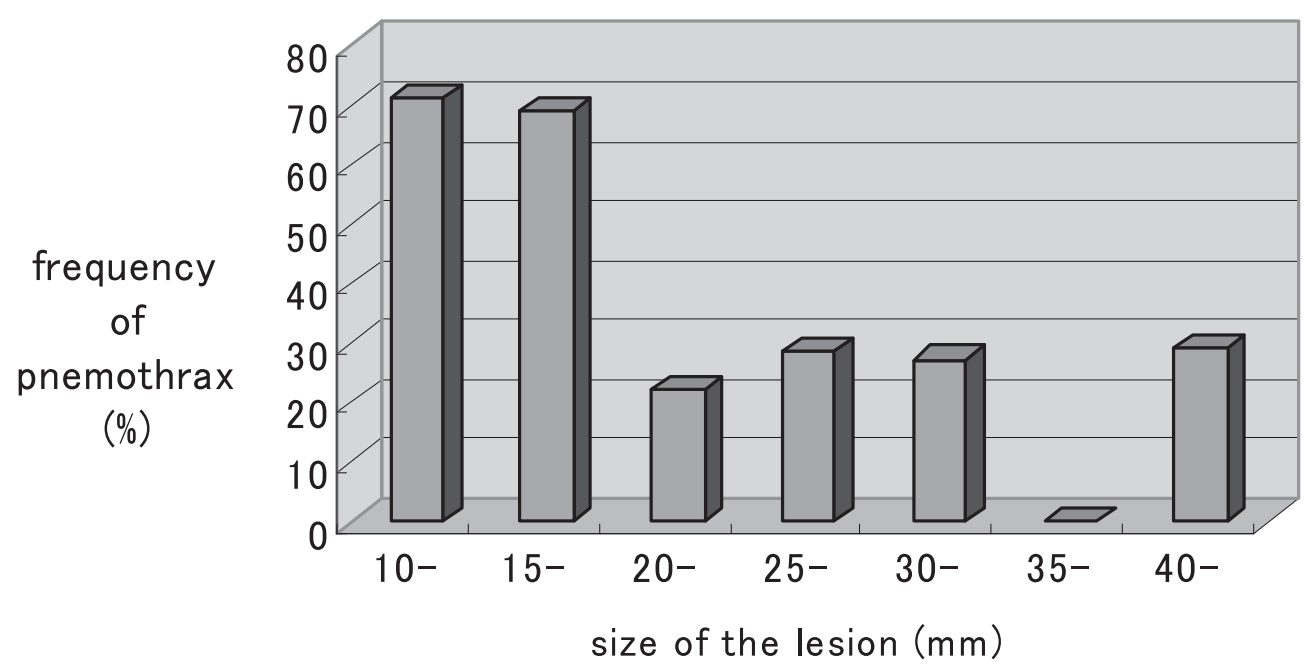

$1:$ Lesion size and frequency of pneumothrax

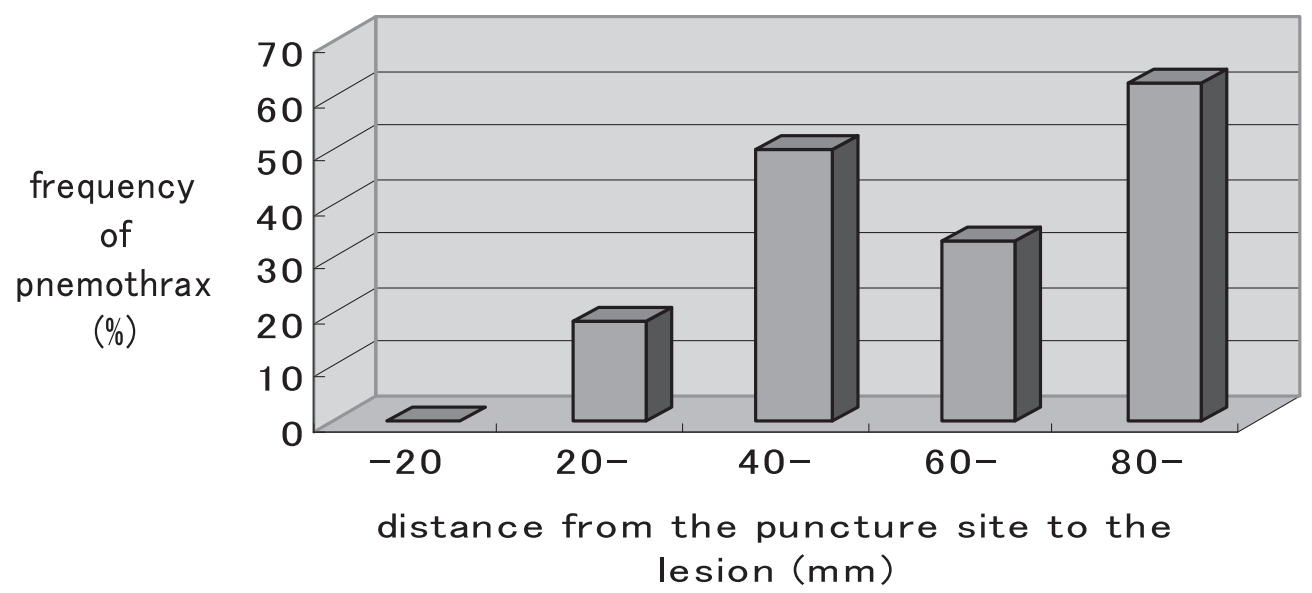

$2:$ Frequency of pnemothrax and distance from puncture site to the lesion

Fig. 3.

\section{Discussion}

The advantages of percutaneous biopsy of the thoracic region using flat detector-equipped CBCT include the convenience and ease of using a work station to plan the 3-dimensional puncture line because $\mathrm{CBCT}$ collects volumetric data, and the versatility of observing the puncture direction from various angles $[\mathrm{Z}$ (body) and $\mathrm{XY}$ axes]. In addition, reflecting the planned puncture angle on the $\mathrm{C}$-arm allows the puncture needle to be arranged on a straight line toward the lesion on fluoroscopy, improving the accuracy of the following lesion puncture ${ }^{4)}$.

Combining the biopsy with fluoroscopy conferred additional advantages to the CBCT 
procedure; 1) enables real-time puncture and avoids the mispuncture of other, nearby structures such as the mediastinum and blood vessels; 2) allows confirmation of the needle tip and puncture angle, thereby increasing the accuracy of biopsy of the target lesion and facilitating a safe operation for both patient and operator; 3) biopsy is performed without confirmation by frequent CT; and, 4) the procedure requires only an angiography room, and does not occupy a CT room used for general diagnosis. As a disadvantage, the use of fluoroscopy increases the exposure level, and the instrument is expensive. Additionally, the image contrast on CBCT is slightly reduced, compared to pure CT. However, biopsy was possible by referring to previous images in all cases. The administration of contrast medium might also improve the image contrast for lesions and blood vessels, if necessary.

The mean time for the biopsy procedure in this study was 56.6 minutes (25-139 minutes). In the case with the longest operation time, the mass was located near the vertebral body directly above the diaphragm. Physiological saline was infused into the extrapleural cavity, and puncture of the mass avoiding the lung field was attempted. However, the lung field was inevitably penetrated because a sufficient space could not be obtained, and a prolonged time was required to complete the puncture.

The mean operation time of CT fluoroscopy-guided biopsy from several studies is 30-60 minutes $^{5-8)}$. Using 3-dimensional data of CBCT thus took a slightly longer time, possibly reflecting the extra data processing required for image collection, with one acquisition taking from 2 minutes and 40 seconds to 4 minutes and 30 seconds. Images were also acquired at least 4 times; this was necessary to ensure the correct puncture position and to confirm the direction of the sheath, sampling site, and potential complications. Data processing after acquisition was likely the main cause of prolonging the operation time, and this will no doubt decrease with future increases in data processing speed.

Several patients in this study experienced pain at the time of puncture, but it was tolerable. Complications of lung biopsy are roughly divided into two groups; those which develop relatively frequently, but are not very problematic clinically, such as pneumothorax and pulmonary hemorrhage, and those which rarely occur but are clinically severe, such as air embolism and the dissemination of malignant cells. Pneumothorax has a high incidence; reportedly about $30 \%$ in CT-guided biopsy ${ }^{9-12)}$, although the incidence of severe cases requiring degassing is low. The incidence of pneumothorax in our patients was 36.4\% $(28 / 77)$, but chest drainage tube placement was necessary in only $9.1 \%(7 / 77)$ of cases, and $3 / 4$ of the events were mild. There was no occurrence of pneumothorax in a lesion that was in contact with the pleura.

Risk factors for pneumothorax include the presence of obstructive pulmonary disorder, distance of the lesion from the chest wall, lesion size, frequency of puncture, operation time, and age. In our patients, the incidence was higher in cases complicated by pulmonary emphysema, with a long distance from the puncture site, small tumor, and long operation time. Hitting the lesion becomes difficult as the lesion size decreases, and the distance 
from the puncture site increases ${ }^{13)}$, increasing the frequency and duration of puncture. These factors may be mutually associated and involved in the development of pneumothorax. The presence of obstructive pulmonary disorder, such as pulmonary emphysema, is also an important factor ${ }^{14,15)}$. Generally, many patients undergoing pulmonary biopsy are elderly or have a cigarette smoking habit, both leading to chronic pulmonary disorder, but lung biopsy cannot be avoided even in the presence of these risk factors ${ }^{16)}$. Operators should therefore accurately recognize the incidence of pneumothorax and use it for preoperative patient counselling and to institute preventive countermeasures ${ }^{17)}$.

Pulmonary hemorrhage occurs with a frequency of 5-15\% and includes alveolar hemorrhage in the lung, blood-stained sputum caused by hemorrhage in the bronchial lumen, and hemoptysis $^{9-12)}$. However, the degree is generally mild, and bleeding stops spontaneously in most cases. In mild alveolar hemorrhage, the bleeding is absorbed within 1-2 days, and no blood-stained sputum or blood loss are detected. In moderate or severe alveolar hemorrhage, a small volume of blood-stained sputum and hemoptysis may be found, but bleeding stops within about 30 minutes $^{18)}$ and no clinical problems eventuate in most cases ${ }^{19)}$. The incidence of pulmonary hemorrhage in our patients was $6.5 \%(5 / 77)$, but no case required special treatment immediately after the procedure.

Air embolism with lung biopsy is a fatal complication and thus clinically important. However, it rarely occurs, with a reported incidence of approximately $0.02 \%{ }^{20)}$. None of the patients in this study developed air embolism. Coughing during lung biopsy can dislocate the biopsy needle tip and cause contact with a vein. Air embolism may therefore occur under a decreased venous pressure immediately before a cough. Antitussive treatment is thus necessary for biopsy patients with coughs. It is also necessary to ensure breath holding when the trocar is removed, and the needle base must be pressed firmly during the removal. In the coaxial method employed at our hospital, a needle one-size larger than the puncture needle is placed at a site close to the lesion, and a thin, longer biopsy needle is inserted into the lesion through the large needle, allowing several specimens to be collected without having to repeatedly puncture the pleura and lung.

Dissemination of malignant cells is another fatal complication associated with biopsy, in which malignant cells are disseminated along the puncture route when the biopsy needle is removed. These disseminated cells might then engraft and proliferate in the lung and/or chest. Dissemination is reportedly very rare, with an incidence of lower than $0.1 \%{ }^{9,11,21)}$, and did not occur in any of our patients. In the coaxial method, the sheath is not inserted into the tumor, and only the trocar is inserted from a site immediately in front of the tumor to collect a specimen, further reducing the possibility of cell dissemination.

Histopathological analysis in the present study revealed malignancy in $90.9 \%$ of lesions biopsied. According to Silit et $a l^{22)}$, CT fluoroscopy-guided percutaneous needle biopsies of the thoracic region are superior to conventional CT-guided biopsy, with adequate material obtained in $85.1 \%$ of cases. Based on the $90.9 \%$ rate of correctly diagnosed malignancy in 
the present study, the described method is at least equally as effective as CT fluoroscopyguided percutaneous needle biopsy. However, we do not expect that all thoracic biopsies will be performed using this technique. Image quality needs to be improved before that is feasible and image data reconstruction needs to be faster. For example, it would be difficult to detect a small, ground-glass attenuation with $\mathrm{CBCT}$ at this time. Further evaluation of this method is clearly warranted, however as is improvement to the CBCT technology.

\section{Conclusion}

Using CBCT equipped with flat panel detector DSA, CBCT- and fluoroscopy-guided needle biopsy could be performed in all patients. Unlike CT-guided biopsy, the procedure can be completed using an angiography room alone, and a reliable biopsy is possible using 3-dimensional data. This percutaneous needle biopsy shows promise for the future.

\section{References}

1) Haaga JR and Alfidi RJ : Precise biopsy localization by computed tomography. Radiology 118 : 603-607 (1976)

2) Katada K, Kato R, Anno H, Ogura Y, Koga S, Ida Y, Sato M and Nonomura K: Guidance with real-time CT fluoroscopy : early clinical experience. Radiology $200: 851-856$ (1996)

3) Kato R, Katada K, Anno H, Suzuki S, Ida Y and Koga S : Radiation dosimetry at CT fluoroscopy: physicians hand dose and development of needle holders. Radiology 201 : 576-578 (1996)

4) Hashimoto $T$, Seino $N$, Honda M, Hayano $D$, Hashizume $T$, Oogiya $Y$, Hirose $M$ and Gokan $T$ : Interventional C-arm CT "DynaCT". Innervision 22(2) : 44-47 (2007) (in Japanese)

5) vanSonnenberg E, Casola G, Ho M, Neff CC, Varney RR, Wittich GR, Christensen R and Friedman PJ : Difficult thoracic lesions: CT-guided biopsy experience in 150 cases. Radiology $167: 457-461$ (1988)

6) Takahashi M, Sano A, Nisizawa S, Murakami M, Ootake S, Fuziwara K, Miki Y, Kawakami K, Kuwata Y, Takada I, Mimura F, Shoda T, Koyama M and Kuroda Y : CT-guided biopsy for thoracic mass lesions followed by fast stain technique. Nippon Acta Radiol $50: 249-257$ (1990) (in Japanese)

7) Shinohara Y: Basic matters needle biopsy of the lung. Mod Physician $15: 237-244$ (1995) (in Japanese)

8) Kadokura M, Tanio N, Nonaka M, Murata N, Yamada M, Funami M, Takaba T and Kushihashi T: CTguided needle biopsy of lung tumors. J Jpn Assoc Chest Surg 5:53-58 (1991) (in Japanese)

9) Sinner WN : Pulmonary neoplasms diagnosed with transthoracic needle biopsy. Cancer $43: 1533-1540$ (1979)

10) Todd TR, Weisbrod G, Tao LC, Sanders DE, Delarue NC, Chamberlain DW, Ilves R, Pearson FG, Cass W and Cooper JD : Aspiration needle biopsy of thoracic lesions. Ann Thorac Surg 32 : 154-161 (1981)

11) Kato H, Kawate N, Yoneyama K and Kinoshita K: Needle biopsy of the lung. Surgery $47: 1382-1385$ (1985) (in Japanese)

12) Saito $T$, Kobayashi H, Danbara $T$, Kira $S$ and Kitamura $S$ : Ultrasonically guided aspiration needle biopsy in the diagnosis of intrapulmonary lesions. J Jpn Respir Soc 26:970-974 (1988) (in Japanese)

13) Shinohara Y: CT-guided needle aspiration biopsy for the diagnosis of intrathoracic lesions. J Jpn Respir Soc 26 : 1052-1061 (1988) (in Japanese)

14) Poe RH, Kallay MC, Wicks CM and Odoroff CL: Predicting risk of pneumothorax in needle biopsy of the lung. Chest $85: 232-235$ (1984)

15) Miller KS, Fish GB, Stanley JH and Schabel SI: Prediction of pneumothorax rate in percutaneous needle aspiration of the lung. Chest $\mathbf{9 3}: 742-745$ (1988)

16) Shinohara Y: The characteristic and indication of CT-guided needle biopsy. Modern Physician $15: 125-132$ (1995) (in Japanese)

17) Hayashi $\mathrm{N}$, Kimoto $\mathrm{T}$, Murashima $\mathrm{H}$, Kimura $\mathrm{H}$, Maeda $\mathrm{M}$, Iwasaki $\mathrm{T}$ and Ishii $\mathrm{Y}$ : Interventional radiology 
of nonvascular sphere. J Med Imaging 11:1313-1324 (1991) (in Japanese)

18) Rock DA : Percutaneous lung biopsy-management of tracheobronchial haemorrhage. Anesthesia 39:888-890 (1984)

19) Berquist TH, Bailey PB, Cortese DA and Miller WE: Transthoracic needle biopsy - accuracy and complication in relation to location and type of lesion. Mayo Clin Proc 55:475-481 (1980)

20) Aberle DR, Gamsu GG and Golden JA : Fatal systemic arterial air embolism following lung needle aspiration. Radiology 165 : 351-353 (1987)

21) Christensen ES: Iatrogenic dissemination of tumor cells-dissemination of tumor cells along the needle track after percutaneous, transthoracic lung biopsy. Dan Med Bull $25: 82-87$ (1978)

22) Silit E, Kizilkaya E, Okutan O, Pekkafali Z, Mutlu H, Cinar Basekim C and Fevzi Karsli A : CT fluoroscopy guided percutaneous needle biopsies in thoracic mass lesions. Eur J Radiol 48 : 193-197 (2003)

[Received January 6, 2009 : Accepted January 26, 2009] 